\title{
A Benchmark Generalization of Fuzzy Soft Ideals in Ordered Semigroups
}

\author{
Faiz Muhammad Khan, Violeta Leoreanu-Fotea, Saifullah, \\ Amanullah
}

\begin{abstract}
In real life, variability and inaccuracy are always presentand must be calculated by either possibilistic, probabilistic, polymorphic or other uncertainty approach. This benchmark study is about to construct new types of fuzzy soft ideals i.e., $\left(\in, \in \vee q_{k}\right)$-FSR(L)Is of ordered semigroup(OSG). Based on this inception, fuzzy soft level subsets are defined which link ordinary ideals with $\left(\in, \in \vee q_{k}\right)$-fuzzy soft left(right) ideals. Some binary operations like $\circ_{\lambda}$, intersection $\cap_{\lambda}$ and union of fuzzy soft sets $\cup_{\lambda}$ are given and various fundamental results of ideal theory are developed through these types of fuzzy soft ideals.
\end{abstract}

\section{Introduction}

Treating global uncertainties in different areas is a major challenge like in, decision making problems, structural engineering, economics, robotics, error correcting codes. Many everyday challenges need a benchmark tools for addressing inaccuracies and uncertainty. Since various research subjects in diverse fields struggle with uncertainty like in geoscience [2], nuclear energy [3], climate predictions [1], geotechnics [10], structural engineering $[4,5,6,7,8,9]$. Numerous hypotheses like interval-valued theory, probability theory, fuzzy sets,

Key Words: Fuzzy soft subsets, OSGs $\left(\in, \in \vee q_{k}\right)$-FSR(L)Is, $\left(\in, \in \vee q_{k}\right)$-fuzzy soft ideals, level subsets

2010 Mathematics Subject Classification: Primary 46G05, 46L05; Secondary 47A30, 47B47.

Received: 26.07 .2020

Accepted: 30.12 .2020 
rough set theory are developed to tackle uncertainty problems. Nevertheless, all these hypotheses are valid and have their significance as well as inherent limitations. The incompatibility of parameters tools is a big issue facing these hypotheses. The benchmark theory of soft set theory was first put in by molodsove [11]; A theory dealing with uncertainty. This new theory was used by the author in smoothness of function, game theory, operational research and theory of probability.This icebreak launch is now used in engineering and decision taking $[12,13,14,15,16,17,18]$, soft integrations, soft derivatives and soft number along with the demands [19] and a number more (also refer $[20,21,22,23,24,25,26,27,28])$. The theory is also used successfully in algebraic structures and various applications are obtained [29, 30, 31, 32, 33, 34]. Because of certain shortcomings in the theory of Zadeh [35] of fuzzy sets The definition of the [36] interval valued sets was introduced providing more appropriate vagueness explanations than fuzzy set.The definition of the fuzzy subgroups was stimulated by Rosenfield [37] while Biswas studied the interval valued fuzzy subgroups [38]. Such hypotheses to a certain degree solve uncertainty issuesYet intrinsic limits still remain due to which Any dynamic problems cannot be adequately answered. The first to offer the idea of a fuzzy soft set and set a certain operation on fuzzy soft set was Maji et al. [39] like; union, Intersection, OR and AND. Most theorists have implemented fuzzy soft sets into diverse algebraic structures. Most of them are; Aygunoglu et al. [40] gave the concept of fuzzy soft set in group theory and looked normal fuzzy soft groups. Feng et al. [41] used fuzzy soft sets and studied soft semirings. The idea of possibility fuzzy soft OSGs was introduced recently by Sana et al. [42] . Bhakat and Das [43] gave the concept of $(\alpha, \beta)$-fuzzy subgroups as they used the relations of $(\in)$ "belongs to" and "quasi-coincident" $(q)$ between a fuzzy subgroup and a fuzzy point which generalizes the Rosenfelds fuzzy subgroup. In BCK/BCI-algebras Jun [44][45] gave the idea of $\left(\in, \in \vee q_{k}\right)$-fuzzy subalgebras and it was also generalize this concept to $\left(\in, \in \vee q_{k}\right)$-fuzzy subalgebras. Shabir et al. [45] further applied this idea of $\left(\in, \in \vee q_{k}\right)$-fuzzy ideals to SGs. The concept of $\left(\in, \in \vee q_{k}\right)$-fuzzy left (right) ideals in OSGs was introduced by Khan et al. [46]. The intent of this study is to incorporate Jun's concept of "generalized quasi coincident with" relation $q_{k}$ and fuzzy soft sets and present new type of fuzzy soft ideal theory i.e., $\left(\in, \in \vee q_{k}\right)$-fuzzy soft ideal in OSGs. Based on this launch, fuzzy soft level subsets are defined. Ordinary ideals and $\left(\in, \in \vee q_{k}\right)$-fuzzy soft ideals are bridged through level sets.Through multiple ideal theory results, different kinds of binary operations like; union of fuzzy soft sets $\cup_{\lambda}, \circ_{\lambda}$ and intersection $\cap_{\lambda}$ are defined and several characterization theorems of OSGs are introduced. 


\section{Preliminaries}

Several basic principles and findings are given in this section which are included in the next part of our developed theory. Begin with the definition of OSG. $(S, \cdot, \leq)$ is known as an OSG if it satisfy the following conditions:

i. $(S, \cdot)$ is a semigroup,

ii. $(S, \leq)$ is a poset,

iii. Left and right compatibility hold in $S$.

$A \neq \emptyset$ we define $A_{b}$ as

$$
A_{b}=\{(x, y) \in S \times S \mid b \leq x y\} .
$$

A fuzzy set $\nu$ of $U$ is a mapping $\psi: U \rightarrow[0,1]$, where $U$ is a non empty universe and $[0,1]$ is usual interval of $\Re$. The set of all fuzzy sets of $U$ is denoted by $\mathcal{F}(U)$. A fuzzy set $\psi$ of $U$ defined as,

$$
\psi(x)= \begin{cases}t, & \text { if } x=y, \\ 0, & \text { otherwise },\end{cases}
$$

is known as fuzzy point with value $t \in(0,1]$ and support $x$ and is denoted by $\frac{x}{t}$. A fuzzy point $\frac{x}{t}$ is said to belong to (resp. quasi-coincident $k$ ) with a fuzzy set $\psi$ and can be written as $\frac{x}{t} \in \psi\left(\right.$ resp. $\left.\frac{x}{t} q_{k} \psi\right)$, where $t \in(0,1]$ and $k$ $\in[0,1)$, unless otherwise stated. For a fuzzy point $\frac{x}{t}$ and a fuzzy set $\psi$ of $U$ we say that:

i. $\frac{x}{t} \in \psi$ if $\psi(x) \geq t$

ii. $\frac{x}{t} q_{k} \psi$ if $\psi(x)+t+k>1$

iii. $\frac{x}{t} \in \vee q_{k} \psi$ if $\frac{x}{t} \in \psi$ or $\frac{x}{t} q_{k} \psi$.

Here we are introducing an ordering relation on $\mathcal{F}(U)$ denoted as $\subseteq_{q_{k}}$ and defined as follows:

For any $\psi, \phi \in \mathcal{F}(U)$, we say $\psi \subseteq_{q_{k}} \phi$ if $\frac{x}{t} \in \psi \Rightarrow \frac{x}{t} \in \vee q_{k} \phi$ for all $x \in U$. Also, $\psi={ }_{q_{k}} \phi$ if $\psi \subseteq_{q_{k}} \phi$ and $\phi \subseteq_{q_{k}} \psi$. if $\alpha \in\left\{\in, q_{k}, \in \vee q_{k}, \subseteq_{q_{k}}\right\}$ then $\bar{\alpha}$ shows us $\alpha$ does not hold, For all $x \in U$ and $I \subseteq U$.

The characteristic function $X_{I}$ is defined as:

$$
X_{I}(x)= \begin{cases}1, & \text { if } x \in I, \\ 0, & \text { otherwise }\end{cases}
$$


Theorem 1. [46] A non empty subset $Y$ of an $O S G S$ is a left (resp. right) ideal of $S$ if and only if the characteristics function $X_{Y}$ of $Y$ is a fuzzy left (resp. right) ideal of $S$.

Let $\psi, \phi \in \mathcal{F}(U)$ then $\forall x \in S$ their product is given as:

$$
(\psi \circ \phi)(x)= \begin{cases}\bigvee_{(a, b) \in A_{x}}\{\psi(a) \wedge \phi(b)\}, & \text { if } \exists(a, b) \in A_{x} \\ 0, & \text { otherwise, }\end{cases}
$$

Let $U$ be a non empty initial universe and $E$ be the parameter set $A \subseteq E$. Then a mapping $\psi: A \rightarrow P(U)$ is called a soft set where $P(U)$ is power set of $U$, soft set is denoted by $(\psi, A)$. A pair $\langle\psi, A\rangle$ is called a fuzzy soft set over $U$ where $\psi$ is a mapping given by $\psi: A \rightarrow \mathcal{F}(U)$. The set of all fuzzy soft sets over $U$ with parameter set $E$ is called a fuzzy soft class and is denoted by $\mathcal{F P}(U, E)$. If $U=\left\{g_{1}, g_{2}, \ldots, g_{m}\right\}$ and $A=\left\{\varepsilon_{1}, \varepsilon_{2}, \ldots, \varepsilon_{n}\right\}$ then the general tabular form of fuzzy soft set can be written as:

$$
\begin{aligned}
\langle\psi, A\rangle=\{ & \left(\psi\left(\varepsilon_{1}\right)\left(g_{1}\right), \psi\left(\varepsilon_{2}\right)\left(g_{1}\right), \ldots, \psi\left(\varepsilon_{n}\right)\left(g_{1}\right), g_{1}\right), \\
\left(\psi\left(\varepsilon_{1}\right)\left(g_{2}\right), \psi\left(\varepsilon_{2}\right)\left(g_{2}\right), \ldots, \psi\left(\varepsilon_{n}\right)\left(g_{2}\right), g_{2}\right), \ldots, & \left.\left(\psi\left(\varepsilon_{1}\right)\left(g_{m}\right), \psi\left(\varepsilon_{2}\right)\left(g_{m}\right), \ldots, \psi\left(\varepsilon_{n}\right)\left(g_{m}\right), g_{m}\right)\right\}
\end{aligned}
$$

Example 1. Bi-polar Disorder.Bipolar disorder is a psychiatric illness which is indicated by severe mood swings from low to high, and from high to low. The low changes are depression and the high ones are mania. These mood changes can be even mixed, that the patient may feel overjoyed and sad simultaneously. Bipolar disorder is difficult to diagnose, Still you should look for any signs. In addition, a patient shows some symptoms minor, A psychologist finds it impossible to determine whether or not a patient has bi-polar disorder. The symptoms of bipolar disorder can be divided into those for mania, and those for depression. A psychologist can use fuzzy soft set for this particular problem to make a decision about four patients i.e. $U=\left\{p_{1}, p_{2}, p_{3}, p_{4}\right\}$. Take the set of symptoms as the set of parameters, corresponding to each parameter psychologist judge the patient and map it to a suitable value in $[0,1]$. Take $E$ is the set parameter i.e. $E=\varepsilon_{1}, \varepsilon_{2}, \ldots, \varepsilon_{1} 4$ where the parameters are given by:

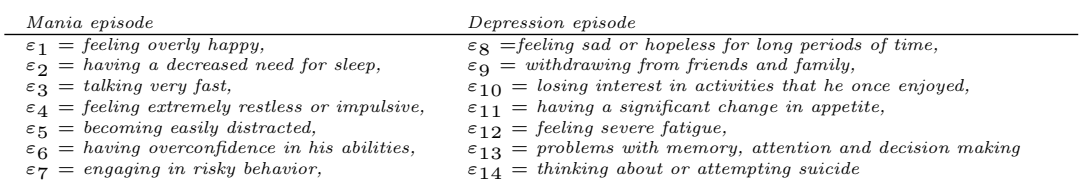

Let $\psi: E \rightarrow \mathcal{F}(U)$ defined in the following table: 
A BENCHMARK GENERALIZATION OF FUZZY SOFT IDEALS IN ORDERED SEMIGROUPS

\begin{tabular}{|c|c|c|c|c|c|c|c|c|c|c|c|c|c|c|}
\hline disorder & \multicolumn{7}{|c|}{ mania episode } & \multicolumn{7}{|c|}{ depression episode } \\
\hline patients & $\psi\left(\varepsilon_{1}\right)$ & $\psi\left(\varepsilon_{2}\right)$ & $\psi\left(\varepsilon_{3}\right)$ & $\psi\left(\varepsilon_{4}\right)$ & $\psi\left(\varepsilon_{5}\right)$ & $\psi\left(\varepsilon_{6}\right)$ & $\psi\left(\varepsilon_{7}\right)$ & $\psi\left(\varepsilon_{8}\right)$ & $\psi\left(\varepsilon_{9}\right)$ & $\psi\left(\varepsilon_{10}\right)$ & $\psi\left(\varepsilon_{11}\right)$ & $\psi\left(\varepsilon_{12}\right)$ & $\psi\left(\varepsilon_{13}\right)$ & $\psi\left(\varepsilon_{14}\right.$ \\
\hline$p_{1}$ & 0.5 & 0 & 0.5 & 0.2 & 0.3 & 0.2 & 0.2 & & 0.2 & & & 0.5 & 0.5 & \\
\hline$p_{2}$ & 0.5 & 0.5 & 0.5 & 0.5 & 0.6 & 0.5 & 0.5 & 0.4 & 0.5 & 0.5 & 0.5 & 0.5 & 0.5 & 0.5 \\
\hline$p_{3}$ & 0.75 & 0.6 & 0.8 & 0.5 & 0.9 & 1 & 1 & 1 & 0.7 & 0.8 & 0.9 & 1 & 0.85 & 0.5 \\
\hline$p_{4}$ & & 1 & 1 & 1 & 1 & 1 & 1 & 1 & 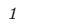 & 1 & 1 & 1 & & 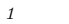 \\
\hline
\end{tabular}

For any patient under consideration during the above operation the sum $\sum_{i=1}^{n} \psi\left(\varepsilon_{i}\right)\left(p_{j}\right), j=1, \ldots m$ Displays the illness stage. In the example above, the aforementioned amount is found for patient $p_{1} 4.1$ Therefore $p_{1}$ has the

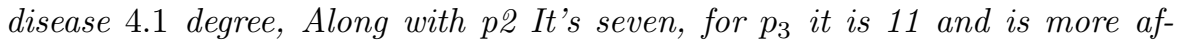
fected as compare to $p_{2}, p_{4}$ has a maximum degree i.e. 14 and is in a serious condition.

$\emptyset \neq I \subseteq S$ is said to be left (resp. right) ideal of $S$ if $\forall x \in S$ and $\forall y \in I, x \leq$ $y \Rightarrow x \in I$ and $S I \subseteq I$ (resp. $I S \subseteq I$ ). A fuzzy soft subset $\langle\psi, A\rangle$ of $S$ is called fuzzy soft left (resp. right) ideal of $S$ if,

i. $\psi(\varepsilon)(x) \geq \psi(\varepsilon)(y)$,

ii. $\psi(\varepsilon)(x y) \geq \psi(\varepsilon)(y)($ resp. $\psi(\varepsilon)(x y) \geq \psi(\varepsilon)(x))$,

for all $x, y \in S$ and $\varepsilon \in A$. A fuzzy soft subset $\langle\psi, A\rangle$ of $S$ is said to be fuzzy soft ideal of $S$ if it is both fuzzy soft left (FSL) and fuzzy soft right ideal (FSRI) of $S$. Let $\langle\psi, A\rangle$ be a fuzzy soft set over $S$ then the level subset is denoted by $U(\langle\psi, A\rangle ; t)$ and is defined as, $U(\langle\psi, A\rangle ; t)=\left\{x \in S \mid \frac{x}{t} \in\langle\psi, A\rangle\right\}$.

Theorem 2. [47] A fuzzy soft subset $\langle\psi, A\rangle$ of an OSG $S$ is fuzzy soft left (resp. right) ideal of $S$ if and only if each non empty level subset $U(\langle\psi, A\rangle, t) \forall t \in(0,1]$ is a left (resp. right) ideal of $S$.

Let $U$ be a universal set and $V \subseteq U$, a fuzzy soft set $\langle\psi, A\rangle$ over $V$ is said to be a relative whole fuzzy soft set (with respect to universe $V$ and parameter set $A)$ denoted by $\Sigma(V, A)$ if $\psi(\varepsilon)=X b_{V} \approx \forall \approx \varepsilon \in A$, we say that $\langle\psi, A\rangle$ is type $\subset_{q_{k}}$ subset of $\langle\phi, B\rangle$ denoted as $\langle\psi, A\rangle \subset_{q_{k}}\langle\phi, B\rangle$ if,

i. $A \subseteq B$

ii. $\psi(\varepsilon) \subseteq \subseteq_{q_{k}} \phi(\varepsilon)$ for any $\varepsilon \in A$.

Moreover, $\langle\psi, A\rangle \asymp_{q_{k}}\langle\phi, B\rangle$ if $\langle\psi, A\rangle \subseteq_{q_{k}}\langle\phi, B\rangle$ and $\langle\phi, B\rangle \subseteq_{q_{k}}\langle\psi, A\rangle$. The product of two fuzzy soft sets is a fuzzy soft set over $S$ denoted by $\langle\psi, A\rangle \odot$ $\langle\phi, B\rangle=\langle\psi \circ \phi, C\rangle$ where $C=A \cup B$ and is defined as,

$$
(\psi \circ \phi)_{\varepsilon}= \begin{cases}\psi(\varepsilon), & \text { if } \varepsilon \in A-B \\ \phi(\varepsilon), & \text { if } \varepsilon \in B-A \\ \psi(\varepsilon) \circ \phi(\varepsilon), & \text { if } \varepsilon \in A \cap B .\end{cases}
$$

Here, $\psi(\varepsilon) \circ \phi(\varepsilon)$ is defined as,

$\psi(\varepsilon) \circ \phi(\varepsilon)(x)= \begin{cases}\bigvee_{(y, z) \in A_{x}}\{\psi(\varepsilon)(y) \wedge \phi(\varepsilon)(z)\} \forall \varepsilon \in A, & \text { if } \exists(y, z) \in A_{x} \\ 0, & \text { otherwise. }\end{cases}$ 


\section{New Generalization of Fuzzy Soft Ideals in Ordered Semi- groups (OSGs)}

In present era, most of the complicated problems involving the global uncertainties are tackle through a benchmark theory i.e., theory of soft sets. The contemporary research in this direction and the new investigations of soft set theory is much productive due to the high level applications of fuzzy soft set theory in the fields of structural engineering, decision making problems, fuzzy automate theory, coding theory and economics. In this segment a new type of fuzzy soft ideal theory based on "generalized quasi coincident with relation $\left(q_{k}\right)$ " is developed. More reliably, $\left(\in, \in \vee q_{k}\right)$-FSL(R)Is of OSG are given and many characterization theorems of OSGs are presented through this new inception. It is important to remember that ordinary ideals are related by characteristic function and level subsets with this new form of fuzzy soft ideals.

Definition 1. A fuzzy soft set $\langle\psi, A\rangle$ over an $O S G S$ is called $\left(\in, \in \vee q_{k}\right)$-fuzzy soft left (resp. right) ideal of $S$ if it satisfies the following conditions:

i. If $x \leq y$ then $\frac{y}{t} \in\langle\psi, A\rangle \Rightarrow \frac{x}{t} \in \vee q_{k}\langle\psi, A\rangle \forall x, y \in S$,

ii. $\Sigma(S, A) \odot\langle\psi, A\rangle \subset_{q_{k}}\langle\psi, A\rangle\left(\right.$ resp. $\left.\langle\psi, A\rangle \odot \Sigma(S, A) \subset_{q_{k}}\langle\psi, A\rangle\right)$.

Moreover, a fuzzy soft set over $S$ is $\left(\in, \in \vee q_{k}\right)$-fuzzy soft ideal if it is both $\left(\in, \in \vee q_{k}\right)$-fuzzy soft left and $\left(\in, \in \vee q_{k}\right)$-FSRI of $S$.

Theorem 3. A fuzzy soft subset $\langle\psi, A\rangle$ of $S$ is an $\left(\in, \in \vee q_{k}\right)$-fuzzy soft left (resp. right) ideal of $S$ if and only if the following conditions hold:

i. $x \leq y \Rightarrow \psi(\varepsilon)(x) \geq \psi(\varepsilon)(y) \wedge \lambda$,

ii. $\psi(\varepsilon)(x y) \geq \psi(\varepsilon)(y) \wedge \lambda($ resp. $\psi(\varepsilon)(x y) \geq \psi(\varepsilon)(x) \wedge \lambda) \forall x, y \in S$, $\forall \varepsilon \in A$ and $\lambda=\frac{1-k}{2}$ unless otherwise stated.

Proof. Let $\langle\psi, A\rangle$ be $\left(\in, \in \vee q_{k}\right)$-fuzzy soft left ideal (FSLI) of $S$. On contrary assume that $\forall x, y \in S, \varepsilon \in A$ and $x \leq y$ where $\psi(\varepsilon)(x)<\psi(\varepsilon)(y) \wedge \lambda$, choose $t \in(0,1]$ such that, $\psi(\varepsilon)(x)<t \leq \psi(\varepsilon)(y) \wedge \lambda$ implies that, $\psi(\varepsilon)(y) \geq t \forall \varepsilon \in A$ thus, $\frac{y}{t} \in\langle\psi, A\rangle$ as, $\psi(\varepsilon)(x)<t$ implies that, $\frac{x}{t} \bar{\epsilon}\langle\psi, A\rangle$. Also, $\psi(\varepsilon)(x)+t+$ $k<\lambda+\lambda+k=1$ implies, $\frac{x}{t} \overline{q_{k}}\langle\psi, A\rangle$. Therefore, $\frac{x}{t} \overline{\in V q_{k}}\langle\psi, A\rangle$ a contradiction to the hypothesis that $\langle\psi, A\rangle$ is an $\left(\in, \in \vee q_{k}\right)$-FSLI. Thus, $\psi(\varepsilon)(x) \geq$ $\psi(\varepsilon)(y) \wedge \lambda$ for all $\varepsilon \in A$. Now, $\left(X_{S}(\varepsilon) \circ \psi(\varepsilon)\right)(x y)=\bigvee_{(p, q) \in A_{x y}}\left\{X_{S}(\varepsilon)(p) \wedge\right.$ $\psi(\varepsilon)(q)\} \geq \bigvee_{(x, y) \in A_{x y}}\left\{X_{S}(\varepsilon)(x) \wedge \psi(\varepsilon)(y)\right\}=1 \wedge \psi(\varepsilon)(y)=\psi(\varepsilon)(y) \geq t$ implies that $\left(X_{S}(\varepsilon) \circ \psi(\varepsilon)\right)(x y) \geq t$ leads to $\frac{x y}{t} \in \Sigma(S, A) \odot\langle\psi, A\rangle$. As $\langle\psi, A\rangle$ is $\left(\in, \in \vee q_{k}\right)$-fuzzy soft ideal, therefore we have $\Sigma(S, A) \odot\langle\psi, A\rangle \subset_{q_{k}}\langle\psi, A\rangle$ implies $\frac{x y}{t} \in\langle\psi, A\rangle$. Now let us assume $\forall x, y \in S, \varepsilon \in A \psi(\varepsilon)(x y)<\psi(\varepsilon)(y) \wedge \lambda$ 
choose $t \in(0,1]$ such that, $\psi(\varepsilon)(x y)<t \leq \psi(\varepsilon)(y) \wedge \lambda$ implies, $\frac{y}{t} \in\langle\psi, A\rangle$, as $\psi(\varepsilon)(x y)<t$ leads to $\frac{x y}{t} \bar{\epsilon}\langle\psi, A\rangle$, Also as, $\psi(\varepsilon)(x y)<t \leq \psi(\varepsilon)(y) \wedge \lambda \leq \lambda$ implies $t \leq \lambda$ means $\psi(\varepsilon)(x y)+t+k<\lambda+\lambda+k=1$ implies $\frac{x y}{t} \overline{q_{k}}\langle\psi, A\rangle$ thus, $\frac{x y}{t} \bar{\vee} q_{k}\langle\psi, A\rangle$ a contradiction to the fact that $\langle\psi, A\rangle$ is an $\left(\in, \in \vee q_{k}\right)$-FSLI. Hence, $\forall \varepsilon \in A, x, y \in S, \psi(\varepsilon)(x y) \geq \psi(\varepsilon)(y) \wedge \lambda$.

Conversely, assume that conditions (i) and (ii) hold. Let $x, y \in S, x \leq y$ and $\frac{y}{t} \in\langle\psi, A\rangle$ i.e., $\psi(\varepsilon)(y) \geq t$ we have, $\psi(\varepsilon)(x) \geq \psi(\varepsilon)(y) \wedge \lambda \psi(\varepsilon)(x) \geq$ $\psi(\varepsilon)(y) \wedge \lambda \geq t \wedge \lambda$ if, $t \leq \lambda$ then $\psi(\varepsilon)(x) \geq t \forall \varepsilon \in A$, implies $\frac{x}{t} \in\langle\psi, A\rangle$. If $t>\lambda$ then, $\psi(\varepsilon)(x) \geq \lambda$ and we have, $\psi(\varepsilon)(x)+t+k \geq \lambda+\lambda+k=1 \forall \varepsilon \in A$. Thus, $\frac{x}{t} q_{k}\langle\psi, A\rangle$, therefore, $\frac{x}{t} \in \vee q_{k}\langle\psi, A\rangle$. Now let $\frac{x y}{t} \in \Sigma(S, A) \odot\langle\psi, A\rangle$. On contrary assume that $\frac{x y}{t} \overline{\in V q_{k}}\langle\psi, A\rangle$ which shows that $\frac{x y}{t} \bar{\epsilon}\langle\psi, A\rangle$ and $\frac{x y}{t} \overline{q_{k}}\langle\psi, A\rangle$ implies, $\psi(\varepsilon)(x y)<t$ and $\psi(\varepsilon)(x y)+t+k \leq 1$. As, $\left(X_{S}(\varepsilon) \circ\right.$ $\psi(\varepsilon))(x y) \geq t$. Since, $x y=x y \Rightarrow(x, y) \in A_{x y}$ therefore, $\left(X_{S}(\varepsilon) \circ \psi(\varepsilon)\right)(x y)=$ $\bigvee_{(x, y) \in A_{x y}}\left\{X_{S}(\varepsilon)(x) \wedge \psi(\varepsilon)(y)=\bigvee_{(x, y) \in A_{x y}}\{1 \wedge \psi(\varepsilon)(y)\}=\psi(\varepsilon)(y)\right.$. Hence, $\psi(\varepsilon)(y) \geq t$ and by condition (ii) we have $\psi(\varepsilon)(x y) \geq \psi(\varepsilon)(y) \wedge \lambda$ implies $\psi(\varepsilon)(x y) \geq \psi(\varepsilon)(y) \wedge \lambda \geq t \wedge \lambda$ if, $t<\lambda$ then, $\psi(\varepsilon)(x y) \geq t \wedge \lambda=t$ leads to $\psi(\varepsilon)(x y)>\lambda$ so $\psi(\varepsilon)(x y)+t+k>\lambda+\lambda+k=1$. Hence, $\frac{x y}{t} q_{k}\langle\psi, A\rangle$, therefore, $\frac{x y}{t} \in\langle\psi, A\rangle$ as we have supposed that $\frac{x y}{t} \in \vee q_{k}\langle\psi, A\rangle$, a contradiction. Thus, $\Sigma(S, A) \odot\langle\psi, A\rangle \subset_{q_{k}}\langle\psi, A\rangle$. Hence, $\langle\psi, A\rangle$ is $\left(\in, \in \vee q_{k}\right)$-FSLI. Similarly, the case for $\left(\in, \in \vee q_{k}\right)$-FSRI can be proved.

Put $k=0$ in Theorem 3, we get the following Corollary.

Corollary 1. A fuzzy soft subset $\langle\psi, A\rangle$ of $S$ is $(\in, \in \vee q)$-fuzzy soft left (resp. right) ideal of $S$ if and only if $\forall \varepsilon \in A$ and $x, y \in S$, the following conditions hold:

i. $x \leq y \Rightarrow \psi(\varepsilon)(x) \geq \psi(\varepsilon)(y) \wedge 0.5$,

ii. $\psi(\varepsilon)(x y) \geq \psi(\varepsilon)(y) \wedge 0.5($ resp. $\psi(\varepsilon)(x y) \geq \psi(\varepsilon)(x) \wedge 0.5)$.

Proof. The proof follows from Theorem 3.

Using Theorem 1 we have the following characterization of fuzzy left (resp. right) ideals of OSGs.

Proposition 1. Let $\emptyset \neq Y \subset S$. Then $Y$ is a left (resp. right) ideal of $S$ if and only if the characteristics function $\left\langle X_{y}, A\right\rangle$ of $Y$ is an $\left(\in, \in \vee q_{k}\right)$-fuzzy soft left (resp. right) ideal of $S$.

Proof. The proof follows from Theorem 3. 
Example 2. Let $S=\{v, w, x, y, z\}$ is an $O S G$ with multiplication table and

ordered relation (hasse diagram) as follows: \begin{tabular}{|c|c|c|c|c|c|}
\hline \hline$\cdot$ & $v$ & $w$ & $x$ & $y$ & $z$ \\
\hline$v$ & $v$ & $y$ & $v$ & $y$ & $y$ \\
$w$ & $v$ & $w$ & $v$ & $y$ & $y$ \\
$x$ & $v$ & $y$ & $x$ & $y$ & $z$ \\
$y$ & $v$ & $y$ & $v$ & $y$ & $y$ \\
$z$ & $v$ & $y$ & $x$ & $y$ & $z$ \\
\hline
\end{tabular}

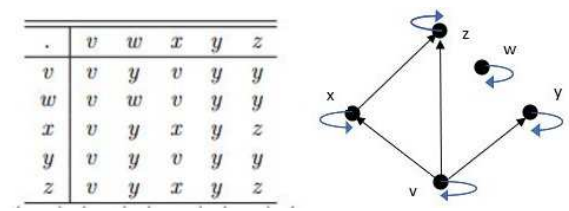

Figure 1: Multiplication table and Ordered relation

\begin{tabular}{c|cc} 
& $\psi\left(\varepsilon_{1}\right)$ & $\psi\left(\varepsilon_{2}\right)$ \\
\hline$v$ & 0.8 & 0.8 \\
$w$ & 0.2 & 0.2 \\
$x$ & 0.7 & 0.8 \\
$y$ & 0.8 & 0.8 \\
$z$ & 0.6 & 0.7
\end{tabular}

Then using Theorem 3, $\langle\psi, A\rangle$ is $\left(\in, \in \vee q_{k}\right)$-fuzzy soft ideal of $S \forall t \in(0, \lambda]$ with $k=0.4$.

Theorem 4. Let I be a left (resp. right) ideal of $S$ and $\langle\psi, A\rangle$ is a fuzzy soft subset of $S$ defined by

$$
\psi(\varepsilon)(x)= \begin{cases}\geq \lambda, & \text { if } x \in I \\ 0, & \text { otherwise }\end{cases}
$$

for all $\varepsilon \in A$. Then, $\langle\psi, A\rangle$ is $\left(\in, \in \vee q_{k}\right)$-fuzzy soft ideal of $S$.

Proof. Let $x \in S, y \in I$ where $x \leq y$ and $t \in(0,1]$ such that $\frac{y}{t} \in\langle\psi, A\rangle$ then $\psi(\varepsilon)(y) \geq t \forall \varepsilon \in A$. Let $I$ be a left ideal of $S$, since, $x \leq y, y \in I$ we have $x \in I$ implies $\psi(\varepsilon)(x) \geq \lambda$. If $t \leq \lambda$, then, $\psi(\varepsilon)(x) \geq t$ leads to $\frac{x}{t} \in\langle\psi, A\rangle$. If $t \geq \lambda$, then $\psi(\varepsilon)(x)+t+k>\lambda+\lambda+k=1$ implies $\frac{x}{t} q_{k}\langle\psi, A\rangle$. Now let $\frac{x}{t} \in \Sigma(S, A) \odot\langle\psi, A\rangle$ we have $\left(X_{S}(\varepsilon) \circ \psi(\varepsilon)\right)(x) \geq t$. Let on contrary suppose that $\frac{x}{\epsilon} \overline{\in q_{k}}\langle\psi, A\rangle$ therefore, $\psi(\varepsilon)(x)<t$ and $\psi(\varepsilon)(x)+t+k \leq 1$ as $\left(X_{S}(\varepsilon) \circ \psi(\varepsilon)\right)(x) \geq t$ implies $\bigvee_{(a, b) \in A_{x}}\left\{X_{S}(\varepsilon)(a) \wedge \psi(\varepsilon)(b)\right\} \geq t$ implies $\bigvee_{(a, b) \in A_{x}}\{1 \wedge \psi(\varepsilon)(b)\} \geq t$. Thus, $\psi(\varepsilon)(b) \geq t$ as $x \in S$ so for $b=x$. Hence, 
$\psi(\varepsilon)(x) \geq t$ implies $\frac{x}{t} \in\langle\psi, A\rangle$ which is a contradiction. Similarly, we can prove $\frac{x}{t} q_{k}\langle\psi, A\rangle$. Thus, $\frac{x}{t} \in \vee q_{k}\langle\psi, A\rangle$, therefor, $\Sigma(S, A) \odot\langle\psi, A\rangle \subset_{q_{k}}\langle\psi, A\rangle$. Hence, $\langle\psi, A\rangle$ is an $\left(\in, \in \vee q_{k}\right)$-FSLI of $S$. Similarly, we can prove $\langle\psi, A\rangle$ is an $\left(\in, \in \vee q_{k}\right)$-FSRI of $S$.

For $k=0$, we get the following Corollary.

Corollary 2. Let I be a left (resp. right) ideal of $S$ and $\langle\psi, A\rangle$ is a fuzzy soft subset of $S$ defined by

$$
\psi(\varepsilon)(x)= \begin{cases}\geq \frac{1}{2}, & \text { if } x \in I \\ 0, & \text { otherwise }\end{cases}
$$

for all $\varepsilon \in A$. Then $\langle\psi, A\rangle$ is an $(\in, \in \vee q)$-fuzzy soft ideal of $S$.

Proof. The proof follows from Theorem 4.

Remark 1. Every fuzzy soft right (resp.left) ideal of an $O S G$ is an $\left(\in, \in \vee q_{k}\right)$ fuzzy soft left (resp. right) ideal of $S$, but the converse is not true as shown in the following example.

Example 3. Consider the OSG given in Example 2 and choose a fuzzy soft set $\langle\psi, B\rangle$ over $S$ defined as follows:

$$
\psi\left(\varepsilon_{1}\right)(a)= \begin{cases}0.8, & \text { if } a=v \\ 0.2, & \text { if } a=w \\ 0.7, & \text { if } a=x \\ 0.5, & \text { if } a=y \\ 0.6, & \text { if } a=z\end{cases}
$$

Then, $\langle\psi, B\rangle$ is an $\left(\in, \in \vee q_{k}\right)$-fuzzy soft ideal, but $U(\langle\psi, B\rangle, t)=\{v, x\}$ for all $t \in(0.6,0.7]$ is not an ideal of $S$. Hence, by Theorem $1,\langle\psi, B\rangle$ is not a fuzzy ideal of $S$ for all $t \in(0.6,0.7]$.

Theorem 5. Every $(\in, \in)$-fuzzy soft ideal is an $\left(\in, \in \vee q_{k}\right)$-fuzzy soft ideal of $S$.

Proof. Let $\langle\psi, A\rangle$ is $(\in, \in)$-FSLI. Let $x, y \in S$ and $x \leq y$ take $\frac{y}{t} \in\langle\psi, A\rangle$. Since, $\langle\psi, A\rangle$ is an $(\in, \in)$-FSLI therefore, $\frac{y}{t} \in\langle\psi, A\rangle$ implies that $\frac{x}{t} \in\langle\psi, A\rangle$, but $\frac{x}{t} \in\langle\psi, A\rangle$ implies $\frac{x}{t} \in \vee q_{k}\langle\psi, A\rangle$. Now let $\frac{x}{t} \in \Sigma(S, A) \odot\langle\psi, A\rangle$. As $\langle\psi, A\rangle$ is an $(\in, \in)$-FSLI therefore, $\frac{x}{t} \in\langle\psi, A\rangle$ since $\frac{x}{t} \in\langle\psi, A\rangle$. Hence, $\frac{x}{t} \in$ $\vee q_{k}\langle\psi, A\rangle$. Thus, $\Sigma(S, A) \odot\langle\psi, A\rangle \subset_{q_{k}}\langle\psi, A\rangle$. Similarly, can be proved for the case of $\left(\in, \in \vee q_{k}\right)$-FSRI of $S$. 
Theorem 6. Every $\left(\in \vee q_{k}, \in \vee q_{k}\right)$-fuzzy soft left (resp. right) ideal is $(\in, \in$ $\vee q_{k}$ )-fuzzy soft left (resp. right) ideal of $S$.

Proof. Suppose $\langle\psi, A\rangle$ is $\left(\in \vee q_{k}, \in \vee q_{k}\right)$-FSLI of $S$. Let $x, y \in S$ where $x \leq y$, take $\frac{y}{t} \in\langle\psi, A\rangle$ implies that, $\frac{y}{t} \in \vee q_{k}\langle\psi, A\rangle$, as $\langle\psi, A\rangle$ is $\left(\in \vee q_{k}, \in \vee q_{k}\right)$ FSLI so $\frac{x}{t} \in \vee q_{k}\langle\psi, A\rangle$. Assume that $\frac{x}{t} \in \Sigma(S, A) \odot\langle\psi, A\rangle$, so by hypothesis $\frac{x}{t} \in \vee q_{k} \Sigma(S, A) \odot\langle\psi, A\rangle$ implies that $\frac{x}{t} \in \vee q_{k}\langle\psi, A\rangle$. Therefore, $\Sigma(S, A) \odot$ $\langle\psi, A\rangle \subset_{q_{k}}\langle\psi, A\rangle$. Hence, $\langle\psi, A\rangle$ is $\left(\in, \in \vee q_{k}\right)$-FSLI of $S$. Similarly, the case for $\left(\in \vee q_{k}, \in \vee q_{k}\right)$-FSRI can also be proved.

Definition 2. Let $\langle\psi, A\rangle$ and $\langle\phi, B\rangle$ be two fuzzy soft subsets of an $O S G$ then $\langle\psi, A\rangle \subset_{\in}\langle\phi, B\rangle$ if $A \subseteq B$ and $\frac{x}{t} \in\langle\psi, A\rangle$ implies $\frac{x}{t} \in\langle\phi, B\rangle$.

Lemma 1. Let $\langle\psi, A\rangle$ and $\langle\phi, B\rangle$ be two fuzzy soft sets over an $O S G S$ such that $\langle\psi, A\rangle \subset \in\langle\phi, B\rangle$ then $\langle\psi, A\rangle \subset_{q_{k}}\langle\phi, B\rangle$.

Proof. The proof is straightforward.

Theorem 7. A fuzzy soft set $\langle\psi, A\rangle$ of $S$ is an $\left(\in, \in \vee q_{k}\right)$-fuzzy soft left (resp. right) ideal of $S$ if and only if every non empty level subset $U(\psi(\varepsilon) ; t)$ is left (resp. right) ideal of $S \forall t \in(0, \lambda]$ and $\varepsilon \in A$.

Proof. Let $\langle\psi, A\rangle$ is $\left(\in, \in \vee q_{k}\right)$-FSLI, let $x \in S, y \in U(\psi(\varepsilon) ; t) \Rightarrow \psi(\varepsilon)(y) \geq t \forall$ $\varepsilon \in A$ by Theorem 1 we have $x, y \in S, x \leq y$ for some $t \in(0, \lambda]$, then $\psi(\varepsilon)(x) \geq$ $\psi(\varepsilon)(y) \wedge \lambda \geq t \wedge \lambda=t$ implies $x \in U(\psi(\varepsilon) ; t)$. Since, $\psi(\varepsilon)(x y) \geq \psi(\varepsilon)(y) \wedge \lambda$ as $\psi(\varepsilon)(y) \geq t$ where, $t \in(0, \lambda]$ therefore, $\psi(\varepsilon)(x y) \geq \psi(\varepsilon)(y) \wedge \lambda \geq t \wedge \lambda=t$. Thus, $x y \in U(\psi(\varepsilon) ; t) \forall \varepsilon \in A$, Hence, $U(\psi(\varepsilon) ; t)$ is a left ideal of $S$.

Conversely, let $U(\psi(\varepsilon) ; t)$ is a left ideal of $S \forall t \in(0, \lambda]$ and assume $x, y \in$ $S, x \leq y$ on contrary suppose that $\psi(\varepsilon)(x)<\psi(\varepsilon)(y) \wedge \lambda$ choose $t \in(0, \lambda]$ such that $\psi(\varepsilon)(x)<t \leq \psi(\varepsilon)(y) \wedge \lambda$ implies $\psi(\varepsilon)(y) \geq t$ so $y \in U(\psi(\varepsilon) ; t)$ as $x \notin U(\psi(\varepsilon) ; t)$ which is a contradiction to the hypothesis. Hence, $\psi(\varepsilon)(x) \geq$ $\psi(\varepsilon)(y) \wedge \lambda$. Let $x, y \in S$, on contrary $\psi(\varepsilon)(x y)<\psi(\varepsilon)(y) \wedge \lambda$ choose $t \in(0, \lambda]$ such that $\psi(\varepsilon)(x y)<t \leq \psi(\varepsilon)(y) \wedge \lambda$, implies $y \in U(\psi(\varepsilon) ; t)$ as $x y \notin U(\psi(\varepsilon) ; t)$ a contradiction to the fact that $U(\psi(\varepsilon) ; t)$ is a left ideal of $S$. Therefore, $\psi(\varepsilon)(x y) \geq \psi(\varepsilon)(y) \wedge \lambda \forall x, y \in S, k \in[0,1)$ and $\forall \varepsilon \in A$. Hence, $\langle\psi, A\rangle$ is an $\left(\in, \in \vee q_{k}\right)$-FSLI. Similarly, we can prove the case of $\left(\in, \in \vee q_{k}\right)$-FSRI.

Proposition 2. A fuzzy set $\langle\psi, A\rangle$ of $S$ is an $\left(\in, \in \vee q_{k}\right)$-fuzzy soft left (resp. right) ideal of $S$. Then, $[\langle\psi, A\rangle]^{\circ}=\{x \in S \mid \psi(\varepsilon)(x)>0\}$ is a left (resp. right) ideal of $S$.

Proof. Let $\langle\psi, A\rangle$ is a fuzzy soft subset of $S$ and is $\left(\in, \in \vee q_{k}\right)$-FSLI of $S$, then $\forall x \in S, y \in[\langle\psi, A\rangle]^{\circ}$ and $x \leq y$ by using Theorem 1 we have $\psi(\varepsilon)(x) \geq$ $\psi(\varepsilon)(y) \wedge \lambda>0 \forall \varepsilon A \rightarrow \psi(\varepsilon)(x)>0 \rightarrow x \in[\langle\psi, A\rangle]^{\circ}$. Also, we have 
$\psi(\varepsilon)(x y) \geq \psi(\varepsilon)(y) \wedge \lambda>0 \rightarrow \psi(\varepsilon)(x y)>0$ implies that $x y \in[\langle\psi, A\rangle]^{\circ}$. Hence, $[\langle\psi, A\rangle]^{\circ}$ is a left ideal of S. Similarly, we can show that $[\langle\psi, A\rangle]^{\circ}$ is a right ideal of $S$.

Lemma 2. Suppose that $\langle\psi, A\rangle$ is an $\left(\in, \in \vee q_{k}\right)$-fuzzy soft left (resp. right) ideal of $S$ such that $\psi(\varepsilon)(x)<\lambda \forall x \in S$ and $\varepsilon \in A$, then $\langle\psi, A\rangle$ is $(\in, \in)$-fuzzy soft left (resp. right) ideal of $S$.

Proof. Let $\langle\psi, A\rangle$ is $\left(\in, \in \vee q_{k}\right)$-FSLI of $S$ such that $\psi(\varepsilon)(x)<\lambda \forall x \in S$. Let $x, y \in S, x \leq y$ and $\frac{y}{t} \in\langle\psi, A\rangle$ implies that $\psi(\varepsilon)(y) \geq t$ and given that $\lambda \geq \psi(\varepsilon)(y)$ implies that $\lambda \geq \psi(\varepsilon)(y) \geq t$ so $\lambda \geq t$. Since, $\psi(\varepsilon)(x) \geq$ $\psi(\varepsilon)(y) \wedge \lambda \geq t \wedge \lambda=t$. Therefore, $\frac{x}{t} \in\langle\psi, A\rangle$. Now as $\Sigma(S, A) \odot\langle\psi, A\rangle \subset_{q_{k}}$ $\langle\psi, A\rangle$ leads $\frac{x}{t} \in \Sigma(S, A) \odot\langle\psi, A\rangle$. So, $\frac{x}{t} \in \vee q_{k}\langle\psi, A\rangle$. If $\frac{x}{t} \in\langle\psi, A\rangle$, then, $\Sigma(S, A) \odot\langle\psi, A\rangle \subset_{\in}\langle\psi, A\rangle$. The case, $\frac{x}{t} q_{k}\langle\psi, A\rangle$ does not hold as $\psi(\varepsilon)(x)<\lambda$ $\forall x \in S$ and $t \leq \lambda$. Therefore, $\psi(\varepsilon)(x)+t+k<\lambda+\lambda+k=1$ implies $\frac{x}{t} \overline{q_{k}}$. Hence, $\langle\psi, A\rangle$ is $(\in, \in)$-FSLI. Similarly, the case for $(\in, \in)$-FSRI can also be proved.

Definition 3. For any fuzzy soft subset $\langle\psi, A\rangle$ of $S$ and $t \in(0,1]$ we denote $Q^{k}(\langle\psi, A\rangle ; t)$ as follows: $Q^{k}(\langle\psi, A\rangle ; t)=\left\{x \in S \mid \frac{x}{t} q_{k}\langle\psi, A\rangle\right\}$ and $[\psi, A]_{t}^{k}=$ $\left\{x \in S \mid \frac{x}{t} \in \vee q_{k}\langle\psi, A\rangle\right\}$. Obviously, $[\psi, A]_{t}^{k}=U(\langle\psi, A\rangle ; t) \cup Q^{k}(\langle\psi, A\rangle ; t)$. $[\psi, A]_{t}^{k}$ is known as $\left(\in \vee q_{k}\right)$-fuzzy soft level subset of $S$ and $Q^{k}(\langle\psi, A\rangle ; t)$ a $q_{k}-$ level fuzzy soft subset of $S$.

Theorem 8. A fuzzy soft subset $\langle\psi, A\rangle$ of $S$ is an $\left(\in, \in \vee q_{k}\right)$-fuzzy soft left (resp. right) ideal of $S$ if and only if $[\psi, A]_{t}^{k}$ is a left (resp. right)-ideal of $S \forall t \in(0,1]$.

Proof. Assume that $\langle\psi, A\rangle$ is an $\left(\in, \vee q_{k}\right)$-FSLI of $S$. Let $x, y \in S, x \leq y$ and $t \in(0,1]$ such that $y \in[\psi, A]_{t}^{k}$ implies $\psi(\varepsilon)(y) \geq t$ or $\psi(\varepsilon)(y)+t+k \geq 1 \forall \varepsilon \in$ $A$. As we have $\psi(\varepsilon)(x) \geq \psi(\varepsilon)(y) \wedge \lambda$. Then the following cases are discussed.

(C1) If $\psi(\varepsilon)(y) \geq t$, then $\psi(\varepsilon)(x) \geq t \wedge \lambda \Rightarrow \psi(\varepsilon)(x) \geq t$ implies that $\frac{x}{t} \in$ $\langle\psi, A\rangle$. If $t>\lambda$, then $\psi(\varepsilon)(x) \geq \lambda$ leads $\psi(\varepsilon)(x)+t+k \geq 1$ so $\frac{x}{t} \in\langle\psi, A\rangle$.

(C2) $\psi(\varepsilon)(x)+t+k>1$, if $t \leq \lambda$, then $\psi(\varepsilon)(x) \geq \psi(\varepsilon)(y) \wedge \lambda \geq 1-t-k=\lambda \geq t$. Which shows that $\frac{x}{t} \in\langle\psi, A\rangle$, if $t>\lambda$, then $\psi(\varepsilon)(x) \geq \psi(\varepsilon)(y) \wedge \lambda>$ $1-t-k \wedge \lambda=1-t-k$ so $\frac{x}{t} q_{k}\langle\psi, A\rangle$. Hence, $\frac{x}{t} \in \vee q_{k}\langle\psi, A\rangle$. Now let $x, y \in S, y \in[\psi, A]_{t}^{k}$. Which leads to $\psi(\varepsilon)(y) \geq t$ or $\psi(\varepsilon)(y)+t+k \geq$ $1 \forall \varepsilon \in A$ therefore, $\psi(\varepsilon)(x y) \geq \psi(\varepsilon)(y) \wedge \lambda \geq t$. Thus, $\frac{x y}{t} \in\langle\psi, A\rangle$.

(C3) $\psi(\varepsilon)(y) \leq t$ if $t \leq \lambda$. Then $\psi(\varepsilon)(x y) \geq t$ leads to $\frac{x y}{t} \in\langle\psi, A\rangle$ if $t>\lambda$. Then, $\psi(\varepsilon)(x y) \geq \lambda$ and so $\psi(\varepsilon)(x y)+t+k \geq \lambda+\lambda+k=1$. Thus, $\frac{x y}{t} q_{k}\langle\psi, A\rangle$. 
(C4) $\psi(\varepsilon)(x y)+t+k>1$ leads to $\frac{x y}{t} q_{k}\langle\psi, A\rangle$. Hence, $\frac{x y}{t} \in \vee q_{k}\langle\psi, A\rangle$, thus $[\psi, A]_{t}^{k}$ is a left ideal of $S$.

Conversely, assume that $\langle\psi, A\rangle$ is a fuzzy soft subset of $S$ such that $[\psi, A]_{t}^{k}$ is a left ideal of $S$ if there exist $x, y \in S, x \leq y, t \in(0, \lambda]$. On contrary assume that $\psi(\varepsilon)(x)<\psi(\varepsilon)(y) \wedge \lambda$ choose $\mathrm{t} \in(0, \lambda]$ such that $\psi(\varepsilon)(x)<t \leq \psi(\varepsilon)(y) \wedge \lambda$, then $\psi(\varepsilon)(y) \geq t$ implies $y \in[\psi, A]_{t}^{k}$ but $x \notin[\psi, A]_{t}^{k}$ a contradiction to the hypothesis. Hence, $\psi(\varepsilon)(x) \geq \psi(\varepsilon)(y) \wedge \lambda$. Now, let $x, y \in S$ such that $\left.f_{(} x y\right)<\psi(\varepsilon)(y) \wedge \lambda$ choose $t \in(0, \lambda]$ such that $\psi(\varepsilon)(x y)<t \leq \psi(\varepsilon)(y) \wedge \lambda$ yield $y \in[\psi, A]_{t}^{k}$ but $\frac{x y}{t} \notin[\psi, A]_{t}^{k}$. A contradiction to the fact that $[\psi, A]_{t}^{k}$ is a left ideal of $S$. Hence, $\psi(\varepsilon)(x y) \geq \psi(\varepsilon)(y) \wedge \lambda \forall x, y \in S, \varepsilon \in A$, thus, $\langle\psi, A\rangle$ is $\left(\in, \in \vee q_{k}\right)$-FSLI of $S$. Similarly, the case for $\langle\psi, A\rangle$ is $\left(\in, \in \vee q_{k}\right)$-FSLI of $S$ can be proved.

Definition 4. Let $(S, \cdot, \leq)$ be an $O S G$ and $\langle\psi, A\rangle,\langle\phi, B\rangle$ are two fuzzy soft sets over $S$, then the $\lambda$-product of $\langle\psi, A\rangle,\langle\phi, B\rangle$ is denoted by $\langle\psi, A\rangle \circ_{\lambda}\langle\phi, B\rangle=$ $\left\langle\psi \circ_{\lambda} \phi, A \cup B\right\rangle$ define as

$$
\left(\psi \circ_{\lambda} \phi\right)(\varepsilon)(x)= \begin{cases}\psi(\varepsilon)(x) \wedge \lambda, & \text { if } \varepsilon \in A-B \\ \phi(\varepsilon)(x) \wedge \lambda, & \text { if } \varepsilon \in B-A \\ \left(\psi(\varepsilon) \circ_{\lambda} \phi(\varepsilon)\right)(x), & \text { if } \varepsilon \in A \cap B\end{cases}
$$

where

$$
\left(\psi(\varepsilon) \circ_{\lambda} \phi(\varepsilon)\right)(x)= \begin{cases}\bigvee_{(y, z) \in A_{x}}\{\psi(\varepsilon)(y) \wedge \phi(\varepsilon)(z) \wedge \lambda\}, & \text { if } A_{x} \neq \emptyset, \\ 0, & \text { otherwise. }\end{cases}
$$

Definition 5. Let $\langle\psi, A\rangle$ and $\langle\phi, B\rangle$ be two fuzzy soft subsets of $S$, then the intersection of $\langle\psi, A\rangle$ and $\langle\phi, B\rangle$ is denoted by $\langle\psi, A\rangle \cap_{\lambda}\langle\phi, B\rangle=\left\langle\psi \cap_{\lambda} \phi, A \cap B\right\rangle$ and defined by:

$$
\left(\psi \cap_{\lambda} \phi\right)(\varepsilon)(x)=\psi(\varepsilon)(x) \wedge \phi(\varepsilon)(x) \wedge \lambda \forall \varepsilon \in A \cap B .
$$

Definition 6. Let $\langle\psi, A\rangle$ and $\langle\phi, B\rangle$ be two fuzzy soft subsets of $S$, then the union of $\langle\psi, A\rangle$ and $\langle\phi, B\rangle$ is denoted by $\langle\psi, A\rangle \cup_{\lambda}\langle\phi, B\rangle=\left\langle\psi \cup_{\lambda} \phi, A \cup B\right\rangle$ and defined by:

$$
\left(\psi \cup_{\lambda} \phi\right)(\varepsilon)(x)= \begin{cases}\psi(\varepsilon)(x) \wedge \lambda, & \text { if } \varepsilon \in A-B \\ \phi(\varepsilon)(x) \wedge \lambda, & \text { if } \varepsilon \in B-A \\ \psi(\varepsilon)(x) \vee \phi(\varepsilon)(x) \wedge \lambda, & \text { if } \varepsilon \in A \cap B\end{cases}
$$


Theorem 9. If $\langle\psi, A\rangle,\langle\phi, B\rangle$ are two $\left(\in, \in \vee q_{k}\right)$-fuzzy soft left (resp. right) ideals of $S$, then $\langle\psi, A\rangle \cap_{\lambda}\langle\phi, B\rangle$ is $\left(\in, \in \vee q_{k}\right)$-fuzzy soft left (resp. right) ideal of $S$.

Proof. Assume that $\langle\psi, A\rangle,\langle\phi, B\rangle$ are $\left(\in, \in \vee q_{k}\right)$-FSLIs of $S$, then for $x, y \in S$ and $x \leq y \psi(\varepsilon)(x) \geq \psi(\varepsilon)(y) \wedge \lambda \forall \varepsilon \in A$ and $\phi(\varepsilon)(x) \geq \phi(\varepsilon)(y) \wedge \lambda \forall \varepsilon \in$ B. Therefore, $\left(\psi(\varepsilon) \cap_{\lambda} \phi(\varepsilon)\right)=\psi(\varepsilon)(x) \wedge \phi(\varepsilon)(x) \wedge \lambda \geq\{\psi(\varepsilon)(y) \wedge \lambda\} \wedge$ $\{\phi(\varepsilon)(y) \wedge \lambda\} \wedge \lambda=(\psi(\varepsilon)(y) \wedge \phi(\varepsilon)(y) \wedge \lambda) \wedge \lambda=\left(\psi(\varepsilon) \cap_{\lambda} \phi(\varepsilon)\right)(y) \wedge \lambda$. Hence, $\left.\left(\psi(\varepsilon) \cap_{\lambda} \phi(\varepsilon)\right)(x) \geq\left(\psi(\varepsilon) \cap_{\lambda} \phi(\varepsilon)\right)(y) \wedge \lambda\right) \forall \varepsilon \in A \cap B$. Let $x, y \in S$ by hypothesis we have $\psi(\varepsilon)(x y) \geq \psi(\varepsilon)(y) \wedge \lambda$ and $\phi(\varepsilon)(x y) \geq \phi(\varepsilon)(y) \wedge \lambda$ as $\left(\psi(\varepsilon) \cap_{\lambda} \phi(\varepsilon)\right)(x y)=\psi(\varepsilon)(x y) \wedge \phi(\varepsilon)(x y) \wedge \lambda \geq\{\psi(\varepsilon)(y) \wedge \lambda\} \wedge\{\phi(\varepsilon)(y) \wedge \lambda\} \wedge \lambda=$ $(\psi(\varepsilon)(y) \wedge \phi(\varepsilon)(y) \wedge \lambda) \wedge \lambda=\left(\psi(\varepsilon) \cap_{\lambda} \phi(\varepsilon)\right)(y) \wedge \lambda$. Hence, $\left\langle\psi(\varepsilon) \cap_{\lambda} \phi(\varepsilon), A \cap B\right\rangle$ is an $\left(\in, \in \vee q_{k}\right)$-FSLI of $S$. Similarly, we can prove that $\left\langle\psi(\varepsilon) \cap_{\lambda} \phi(\varepsilon), A \cap B\right\rangle$ is an $\left(\in, \in \vee q_{k}\right)$-FSRI of $S$.

\section{Conclusion}

The new investigations using soft structures in applied fields like structural engineering and decision making problems are becoming the central focus for researchers. This benchmark piece of research achieved another milestone in the ideal theory of OSGs by developing fuzzy soft ideal theory in OSGs. More precisely, we introduced $\left(\in, \in \vee q_{k}\right)$-fuzzy soft ideals of OSGs and determined several important characterization theorems of OSGs based on this new inception. Also ordinary ideals and fuzzy soft ideals of type $\left(\in, \in \vee q_{k}\right)$ are linked through characteristic functions and level subsets. Further, this theory will provide a suitable platform for further research i.e., $\left(\in, \in \vee q_{k}\right)$-fuzzy soft biideal (resp. interior, generalized bi, quasi)-ideals of an OSG will be developed.

\section{Acknowledgment}

This research is the part of NRPU project 6831 by HEC. The first author would like to acknowledge Higher Education Commission (HEC) for NRPU project 6831 .

\section{References}

[1] J. Slingo and T. Palmer, Uncertainty in weather and climate prediction. Philosophical Transactions of the Royal Society A Mathematical, Physical and Engineering Sciences, 369:4751 - 4767, 2011.

[2] S. J. Fletcher, Data Assimilation for the Geosciences From Theory to Application. Elsevier, 2017. 
[3] F. Bostelmann and G. Strydom. Nuclear data uncertainty and sensitivity analysis of the vhtrc benchmark using scale. Annals of Nuclear Energy, $110,317-329,2017$.

[4] A. Csebfalvi, Structural optimization under uncertainty in loading directions: Benchmark results. Advances in Engineering Software, 120:68 -78, 2018.

[5] T. Gernay, R. V. Coile, N. E. Khorasani and D. Hopkin, Efficient uncertainty quantification method applied to structural fire engineering computations. Engineering Structures, 183:1 - 17, 2019.

[6] H. Zhai and J. Zhang, Equilibrium reliability measure for structural design under twofold uncertainty. Information Sciences, 477:466 - 489, 2019

[7] Y. Zhang, Y. Lu, Y. Zhou and Q. Zhang, Resistance uncertainty and structural reliability of hypar tensioned membrane structures with pvc coated polyesters. Thin-Walled Structures, 124:392 - 401, 2018.

[8] M. Guedri, S. Cogan and N. Bouhaddi, Robustness of structural reliability analyses to epistemic uncertainties. Mechanical Systems and Signal Processing, 28:458 - 469, 2012.

[9] G.Schueller and H.Pradlwarter, Benchmark study on reliability estimation in higher dimensions of structural systems an overview. Structural Safety, 29(3):167-182, 2007.

[10] M. Huber, Reducing forecast uncertainty by using observations in geotechnical engineering. Probabilistic Engineering Mechanics, 45:212219,2016

[11] D.Molodtsov, Soft set theory-first results, Comput. Math. Applic. 37(4), 19-31 (1999).

[12] F.Feng, Soft rough sets applied to multicriteria group decision making, Ann. Fuzzy Math. Inform., 2, 69-80,(2011)

[13] P.K.Maji, A.R.Roy and R.Biswas, An application of soft sets in a decision making problem, Comput. Math. Applic., 44, 1077-1083,(2002).

[14] A.R.Roy and P.K.Maji, A soft set theoretic approach to decision making problems, Journal of Computational and Applied Mathematics 203,412418.(2007).

[15] N.cagman and S.Enginoglu, Soft matrix theory and its decision making, Comput. Math. Appl. 59, 3308-3314.(2010). 
[16] N.cagman and S.Enginoglu, Soft set theory and uni-int decision making, Eur. J. Oper. Res. 207, 848-855.(2010).

[17] F.Feng, Y.B.Jun, X.Liu and L.Li, An adjustable approach to fuzzy soft set based decision making, J. Comput. Appl. Math. 234, 10-20.(2010).

[18] P.K.Maji, A.R.Roy and R.Biswas, An application of soft sets in a decision making problem, Comput. Math. Appl. 44, 1077-1083.(2002).

[19] D.Molodtsov, V.Yu.Leonov and D.V.Kovkov, Soft set technique and its applications, Nechetkie Sistemy i Myagkie Vychisleniya, 1(1), 8-39.(2006).

[20] U.Acar, F.Koyuncu and B.Tanay, Soft sets and soft rings, Comput. Math. Appl., 59, 3458-3463.(2010).

[21] A.Sezgin and A.O.Atagun, On operations of soft sets, Comput. Math. Appl., 61, 1457-1467.(2011).

[22] N.Cagman and S.Enginoglu, FP-soft set theory and its applications, Ann. Fuzzy Math. Inform., 2, 219-226.(2011).

[23] F.Feng, X.Y.Liu, V.Leoreanu-Fotea and Y.B.Jun, Soft sets and soft rough sets, Inform. Sci., 181, 1125-1137.(2011).

[24] Z.Xiao, K.Gong, S.Xia and Y.Zou, Exclusive disjunctive soft sets, Comput. Math. Appl., 59, 2128-2137.(2010).

[25] Y.Zou and Z.Xiao, Data analysis approaches of soft sets under incomplete information, Knowledge Based Systems, 21, 941-945.(2008).

[26] A.O.Atagun and A.Sezgin, Soft substructures of rings, fields and modules, Comput. Math. Appl., 61, 592-601.(2011).

[27] F.Feng, Y.B.Jun and X.Zhao, Soft semirings, Comput. Math. Appl. 56, 2621-2628.(2008).

[28] P.Majumdar and S.K.Samanta, Similarity measure of soft sets, New Mathematics and Natural Computation, 4(1), 1-12.(2008).

[29] Y. B.Jun, Soft BCK/BCI-algebras. Comput. Math. Applic., 56, 14081413.(2008).

[30] Y. B.Jun, K.J.Lee and A.Khan, Soft OSGs. Math.Logic Q., 56, 4250.(2010).

[31] Y. B.Jun, K.J.Lee and C.H.Park, Soft set theory applied to ideals in d-algebras. Comput. Math. Applic., 57, 367-378.(2009). 
[32] Y. B.Jun, K.J.Lee and J.Zhan, Soft p-ideals of soft BCI-algebras, Comput. Math. Applic., 58, 2060-2068.(2009).

[33] Y. B.Jun and C.H.Park, Applications of soft sets in ideal theory.,Inform. Sci., 178, 2466-2475.(2008).

[34] J.Zhan and Y. B.Jun, Soft BL-algebras based on fuzzy sets., Comput. Math. Applic., 59, 2037-2046.(2010).

[35] L. A. Zadeh, Fuzzy sets., Inform. Control. 8, 338-353 (1965).

[36] L. A. Zadeh, The concept of a linguistic variable and its application to approximate reasoning., Inform. Sci. 8, 199-249 (1975).

[37] A.Rosenfeld, Fuzzy groups., J. Math. Anal. Appl. 35, 512-517 (1971).

[38] R.Biswas Rosenfelds, fuzzy subgroups with interval-valued membership functions., Fuzzy Sets and Systems volume. 63, 87-90 (1994).

[39] P. K. Maji, R. Biswas, A. R. Roy, Fuzzy soft sets, J. Fuzzy. Math. 9(3), 589-602 (2001).

[40] A. Aygunoglu, H. Aygun Introduction to fuzzy soft group Coumpt. Math. Appl. 58, 1279-1286 (2009)

[41] Feng Feng, Young Bae Jun, Xianzhong Zhao Soft semirings, Computers and Mathematics with Applications. 56, 2621-2628 (2008)

[42] Sana. H, F. M. Khan, N. Yufeng, A new concept of possibility fuzzy soft OSGs via its applications, J. Int. Fuzzy Sys. 36, 3685-3696 (2019).

[43] S. K. Bhakat, P. Das $(\in, \in \vee q)$-fuzzy subgroups, Fuzzy Sets and Systems. 80, 359-368 (1996).

[44] Y. B. Jun, Generalizations of $(\in, \in \vee q)$-fuzzy subalgebras in BCK/BCIalgebras, Comput. Math. Applic. 58(7), 1383-1390 (2009).

[45] M. Shabir, Y. B. Jun, H. Nawaz, Semigroups characterized by $\left(\in, \in \vee q_{k}\right)$ -fuzzy ideals, Comput. Math. Applic. 60, 1473-1493 (2010).

[46] F. M. Khan, N. H. Sarmin, A. Khan, Some study of $(\alpha, \beta)$-fuzzy ideals in OSGs, Anal. Fuzzy. Math. Inform. 3(2), 213-227 (2012).

[47] Cheng-Fu Yang,Fuzzy soft semigroups and fuzzy soft ideals,Computers and Mathematics with Applications 61 (2011) 255-261 
A BENCHMARK GENERALIZATION OF FUZZY SOFT IDEALS IN ORDERED SEMIGROUPS

Faiz Muhammad Khan,

Department of Mathematics and Statistics,

University of Swat,

Khyberpakhtunkhwa, Pakistan.

Email: faiz_zady@yahoo.com

Violeta Leoreanu-Foteab,

Department of Mathematics,

Al. I. Cuza University of Iasi,

Bd Carol I, no 11 Romania.

Email: foteavioleta@gmail.com

Saifullah,

Department of Mathematics,

University of Malakand,

Lower Dir, Khyberpakhtunkhwa, Pakistan.

Email: saifullah9090@hotmail.com

Amanullah,

Department of Mathematics,

University of Malakand,

Lower Dir, Khyberpakhtunkhwa, Pakistan.

Email: amanswt@uom.edu.pk 